\title{
METODE.Bach': MÉTODO PARA LA TOMA DE DECISIONES ACADÉMICAS Y PROFESIONALES EN EL BACHILLERATO
}

\author{
METODE.Bach: METHOD FOR EDUCATIONAL AND VOCATIONAL \\ DECISIONS MAKING WITHIN BACHILLERATO
}

\author{
José A. Benavent*, Francisco Bayarri**, Julián García***, \\ Luis Ignacio Ramírez****, Santiago Vivo***** \\ Miembros del equipo CESOF
}

\section{RESUMEN}

METODE.Bach constituye un método completo y eficaz para la toma de decisiones en el Bachillerato. Se presenta en formato texto (lápiz y papel) y en CD-Rom interactivo. El formato texto está diseñado para aplicarse en dos sesiones de 60 minutos supervisadas por el tutor. Si se aplica de forma interactiva, las sesiones pueden organizarse a criterio del sujeto. El Método ha sido contrastado y validado interna y externamente. Permite una toma de decisiones con garantía de éxito, facilita el intercambio de opiniones en el seno familiar y se convierte en un instrumento motivador de las tareas propias de la orientación y la tutoría.

Palabras clave: Orientación escolar y profesional, toma de decisiones, Bachillerato, plan de acción tutorial (PAT), método autoaplicable de orientación académica y profesional.

1. Proyecto I+D en el marco del Plan Nacional de Investigación Científica, Desarrollo e Innovación Tecnológica 2000-2003, cofinanciado por el Ministerio de Ciencia y Tecnología y FEDER, y con número de referencia BSO2003-09667/PSCE.

* José A. Benavent. Dr. Filosofía y Letras (Pedagogía) y Master en Counseling and Guidance por la New York University (USA). Prof. titular jubilado de la Universidad de Valencia y Presidente de AVOP.

** Francisco Bayarri. Dr. Pedagogía, Prof. asociado Dto. Pedagogía General.Univ. Valencia y Orientador IES "Massamagrell".

*** Julián García. Dr. Psicología. Prof. asociado Dto. Ps. Evolutiva y de la Educación. Univ. Valencia y Orientador del IES “Quart de Poblet”.

**** Luis Ignacio Ramírez. Dr. Psicología y Orientador de SPE de la Consejeria de Educación de la Generalidad Valenciana.

***** Santiago Vivo. Licenciado en Pedagogía y Prof. tutor IES “Cid Campeadoor” de Valencia.

Miembros del equipo CESOF cuya línea de investigación preferente es la Orientación académica y la toma de decisiones en Educación Secundaria. Contacto: Jose.Benavent@uv.es. 


\begin{abstract}
METODE.Bach is a powerful comprehensive tool for decisions making within Bachillerato (postcompulsory secondary school). It appears in printed format (pencil and paper) and in electronic format, as an interactive CD-Rom. The first one has been designed for its use throughout two tutorised 60 minute long sessions. If applied in an interactive way, it allows the student free management of the work sessions The Method has been assessed and validated, both internally and externally. It provides the student with a quality decisions making method, which is very likely to be successful. It also improves personal opinion exchanges within the family and becomes a motivating tool for school guidance.
\end{abstract}

Key words: Educational and vocational guidance, decisions making, Bachillerato, tutorial action plan (TAP), self-applied method for educational and vocational guidance.

\title{
Introducción
}

METODE.Bach pretende ser un eficaz instrumento para facilitar la toma de decisiones académicas y profesionales de los alumnos de Bachillerato. Destreza que tendrán que desarrollar a lo largo de los dos cursos de duración que actualmente tiene este nivel educativo del vigente sistema escolar español.

Nuestro método es oportuno y necesario para la información y asesoramiento del alumnado en estos momentos decisivos de su formación, pues viene a llenar un vacío existente en la orientación académica y profesional de los escolares de 16 a 18 años en el Bachillerato LOGSE, así como en las reformas que se producirán con la progresiva implantación de la Ley Orgánica de Educación (MEC, 2006). METODE.Bach, está diseñado para que pueda autoaplicarse individualmente, o trabajarse en el grupo de alumnos, e integrarse en el PAT de los IES y los centros de educación secundaria privados como una actividad más de la acción tutorial ordinaria.

Nuestro método se inscribe en la corriente teórica que fundamenta la Orientación escolar y profesional en el tratamiento cognitivo de la información. Desde este posicionamiento, la Orientación la entendemos como un proceso educativo en el que, con el asesoramiento del orientador y/o el profesor-tutor, el aprendizaje que realiza el alumno sobre si mismo, su medio y los itinerarios académicos y profesionales que se le ofertan, contribuye al desarrollo de la significación y comprensión de la realidad escolar y laboral facilitando la elección y la toma de decisiones responsables. Dicho aprendizaje se produce en la medida en que se actúa gracias al autocontrol que hace el aprendiz de sus viejos conocimientos para la solución de los nuevos problemas o "aprendizaje ambiental reactivo" (Duchastel, 1986). Este enfoque conceptual supone:

$1^{\circ}$ ) Disponer de recursos informativos adaptados a las características de los sujetos sobre las principales áreas cognitivas implicadas en la elección y

$2^{\circ}$ ) Diseñar un paradigma adecuado para la solución de problemas y la toma de decisiones.

Entre los numerosos modelos cognitivos que cumplen estos requisitos hemos elegido el desarrollad en los EE.UU. por la Florida State University (Peterson et al., 1991). Modelo base que describimos y adaptamos al contexto educativo español en un trabajo anterior (Benavent, 1994), y que utilizamos con éxito en el diseño, construcción y validación del programa CESOF para la toma de decisiones al finalizar la ESO (Benavent et al., 
2000, 2000a, 2001 y 2002). Nuestro paradigma tiene en cuenta las siguientes dimensiones cognitivas:

1) Autoconocimiento: reflexión sobre las propias aptitudes, capacidades. sentimientos y valores.

2) Conocimiento y comprensión de las opciones académicas y profesionales a las que tiene acceso el alumnado al finalizar la educación secundaria post-obligatoria (Bachillerato).

3) Comprensión de sí mismo en relación con sus opciones.

4) Conocimiento y comprensión de la situación social, económica y cultural en las que está inmerso el alumno y su familia.

5) Conocimiento, comprensión y desarrollo de destrezas para la toma de decisiones y

6) Metacognición: reflexión sobre la toma de decisiones y convicción de que se ha tomado una decisión coherente y responsable.

Para transformar este paradigma teórico, en un modelo de intervención eficaz que responda a las características y necesidades del alumnado de Bachillerato, nuestro equipo ha realizado un estudio crítico, exhaustivo y riguroso de los principales métodos, programas y procedimientos autoaplicables generados en España sobre orientación profesional y toma de decisiones de los alumnos al finalizar la educación secundaria en los anteriores planes de estudio de BUP y COU, y en algunos casos, de sus posteriores adaptaciones al nuevo Bachillerato LOGSE (Álvarez y Fernández, 1987 y 1996; Álvarez Rojo, 1991; Bisquerra, 1989; Corominas, 1996; De la Cruz, 1993 y 1997; García Mediavilla et al., 1990; Martínez y Valls, 1999; Repetto, 1999; Rivas et al., 1989 y 1998; Rodríguez Moreno, 1994; Yuste, 1988 y 2001). También se han tenido en cuenta los últimos trabajos realizados sobre evaluación de los intereses básicos académico profesionales y sobre las preferencias hacia los ciclos formativos de los alumnos de educación secundaria (Hernández Franco, 2004; Hernández Franco et al., 2004) Además, hemos analizado la problemática escolar y el clima institucional de una muestra de centros que imparten Bachillerato en la conurbación de la ciudad de Valencia y entrevistado a sus profesores-tutores y orientadores, así como las características sociales, culturales, económicas y laborales del medio en que están inmersos los alumnos y sus familias, a fin de adecuar nuestro método a las necesidades observadas y manifestadas y, propiciar en lo posible, el logro de los siguientes objetivos:

a) Facilitar al alumno información actualizada y suficiente sobre las características del sistema educativo y el mundo del trabajo que afectan directamente el momento de transición en que se encuentra y que condicionan su toma de decisiones.

b) Favorecer el desarrollo de estrategias y habilidades para la toma de decisiones.

c) Posibilitar el ajuste y objetivación del autoconcepto para una adecuada valoración de las opciones académicas y socio-laborales que se le presentan al finalizar el Bachillerato.

d) Concienciar la importancia de controlar y dirigir la propia toma de decisiones y

e) Reforzar una toma de decisiones coherente y responsable con el apoyo de la familia. 


\section{Método}

Nuestra investigación se ha desarrollando, a lo largo del trienio 2003-2006, temporalizada en tres anualidades, y en estos momentos, diciembre de 2007 damos por finalizado el trabajo.

A lo largo de este proceso hemos utilizando distintas estrategias, técnicas, instrumentos y pruebas propias de la metodología cuantitativa y cualitativa de las Ciencias Sociales para el tratamiento de datos y su análisis crítico y estadístico. Para la construcción de los cuestionarios hemos utilizado una escala Likert con cuatro ó cinco posibilidades de respuesta, según los casos. Los datos recogidos al aplicar los cuestionarios en la prueba piloto han sido sometidos a un análisis factorial por el método de extracción de componentes principales y de rotación por normalización Varimax con Kaiser, mientras que la consistencia interna de dichos cuestionarios se ha determinado mediante el cálculo del coeficiente $\alpha$ de Cronbach. La toma de decisiones se facilita por medio de una estrategia original creada por el equipo investigador que combina e interpreta los datos que se van acumulando y arrastrando a lo largo del proceso. La validación externa de METODE.Bach se determina por el grado de satisfacción expresado por alumnos, padres, profesores tutores y orientadores que han participado en la elaboración de nuestro método por medio de sus respuestas a unos cuestionarios específicos que hemos construido para este propósito.

\section{Diseño}

Nuestra investigación, en función de los objetivos que hemos pretendido alcanzar, es un estudio mixto empírico experimental ex-post-facto y responde a una estrategia de tipo transversal en la que se han utilizado cuatro muestras: dos, del universo de estudiantes de Bachillerato LOGSE de la Comunidad Valenciana, otra, de sus familias, y la cuarta, de los profesores tutores y orientadores de los centros educativos implicados en el proceso. Además, tras un análisis exhaustivo y riguroso de los programas de orientación profesional existentes en el mercado, de analizar la problemática escolar de los centros que imparten Bachillerato en nuestro territorio y de ajustarnos a los fundamentos científicos expresados, decidimos diseñar una herramienta funcional que reuniera las siguientes características:

a) Facilidad de comprensión.

b) Sencillez manipulativa de materiales e instrumentos.

c) Economía de recursos y

d) Máxima adecuación a las características personales, familiares y del contexto ecológico del alumnado.

\section{Muestras}

Para la aplicación experimental y validación interna de nuestro Método, hemos contado con dos muestras incidentales de carácter no probabilístico de alumnos de primero y segundo curso de los Bachilleratos LOGSE. La primera muestra, utilizada en la prueba piloto, está compuesta por un total de 90 casos válidos $(\mathrm{N}=90)$, de los que 57 son mujeres y 43 
varones y proceden de tres IES y un colegio privado de la provincia de Valencia. La segunda muestra, empleada en la aplicación experimental del Método, consta de un total bruto de 1.000 alumnos, de los que se han recogido 967 casos, resultando finalmente válidos 948, de los que 403 estaban en $1^{\circ}$ curso y los 545 restantes en $2^{\circ}$ curso, pertenecientes a 14 IES y 4 colegios privados con ofertas no concertadas de Bachillerato, todos ellos de la Comunidad Valenciana (Castellón, Valencia y Alicante). De esta muestra, el 52,20\% son mujeres y el $47,80 \%$ varones. Para su validación externa se han utilizado tres muestras: una de alumnado, de los 948 sujetos que contestaron correctamente los cuestionarios se seleccionaron por sorteo estratificado al azar entre los colegios e IES participantes un total de 400 alumnos de los que se recogieron 372 casos válidos; otra, de 206 casos válidos entre los padres de los 372 alumnos seleccionados, y finalmente, una tercera muestra de 19 turotes/orientadores de los centros educativos participantes.

\section{Variables}

Para dar cumplimiento a los objetivos propuestos y ceñirnos a las características de funcionalidad que acabamos de exponer, consideramos que debíamos de disponer, como mínimo, de información significativa sobre las siguiente variables:

- Resultados académicos. Calificaciones correspondientes a los expedientes académicos de los alumnos en el momento de la aplicación del Método.

- Áreas vocacionales preferentes. Definidas como primera y segunda área de preferencia, en función de las puntuaciones ajustadas y jerarquizadas obtenidas con la aplicación del Cuestionario de Preferencias Profesionales (CPP), entre las 17 áreas recogidas en el mismo: científica, técnica, económico-empresarial, ciencias de la naturaleza, agropecuaria, biosanitaria, ocio, profesionales del deporte, humanidades, psicopedagógica, sociológica, derecho y asesoramiento, comunicación, seguridad, artes escénicas, música y artes plásticas.

- Condicionantes de la toma de decisiones. Definidos como generales, personales y del estudio, en función del ajuste dimensional de los resultados obtenidos con el pase del Cuestionario de condicionantes de la toma de decisiones $(\mathrm{Q})$, entre las 7 dimensiones contempladas en el mismo: económica, familiar, geográfica, de la salud, personal, hábitos de estudio y rendimiento académico.

- Grado de satisfacción tras la aplicación del Método. Expresada por las tres poblaciones participantes, alumnado, padres y tutores/orientadores, sobre: la utilidad funcional y eficacia del Método, la pertinencia de su formato y estructura, la información aportada y la adecuada toma de decisiones.

\section{Instrumentos}

Atendiendo a los principios y fundamentos científicos y criteriales expuestos anteriormente se han diseñado, construido y validado los siguientes instrumentos:

- Ocho rejillas para la tabulación de las notas académicas del alumno en el momento de la aplicación del Método. Dos rejillas, una para primero y otra para segundo 
curso, por cada una de las modalidades del actual Bachillerato LOGSE (Ciencias de la Naturaleza y la Salud, Tecnología, Humanidades y Ciencias Sociales, y Artes).

- Cuestionario de Preferencias Profesionales (CPP). El CPP tiene como marco teórico de referencia los "intereses básicos" y la línea trazada por las investigaciones anglosajonas y su literatura científica sobre escalas de intereses profesionales y contempla 17 áreas vocacionales de intereses profesionales básicos en 182 items con cinco posibilidades de respuesta (Likert), en los que el encuestado expresa su agrado o desagrado con las profesiones y actividades profesionales propuestas. Los perfiles obtenidos, a partir de las puntuaciones alcanzadas por un sujeto, nos muestran las preferencias relativas de una área respecto a las restantes.

- Cuestionario de Condicionantes de la toma de decisiones (Q). El cuestionario Q, con sus 35 items, es el resultado de la síntesis de una batería de siete cuestionarios de 30 items cada uno (total 210 items), de los que por razones de funcionalidad y economía de tiempos, se seleccionaron, después de la prueba piloto, los 5 más significativos de cada cuestionario y que nos aportan información sensible sobre las siguientes siete condicionantes de la toma de decisiones:

1) Económicos.

2) Familiares.

3) Geográficos.

4) De la salud.

5) Personales.

6) Hábitos de estudio y

7) Rendimiento académico

- Tres cuestionarios para la validación externa del Método tras su aplicación. Para contrastar la validez externa del Método hemos construido tres cuestionarios con cuatro posibilidades de respuesta (Likert), para recoger las opiniones del alumnado (20 items), padres (12 items) y profesores tutores/orientadores (14 items) una vez finalizado el proceso de aplicación, emisión del consejo orientador y la toma de decisiones por parte del alumno y su familia.

\section{Resultados}

Finalizado nuestro trabajo se han obtenido los siguientes resultados en relación con los objetivos propuestos:

Construcción de ocho rejillas para la tabulación de las notas académicas del alumno en el momento de la aplicación del Método. Dos rejillas, una para primero y otra para segundo curso, por cada una de las modalidades del Bachillerato LOGSE (Ciencias de la Naturaleza y la Salud, Tecnología, Humanidades y Ciencias Sociales, y Artes), dado que los resultados académicos son buenos indicadores o predictores de posteriores rendimientos escolares y/o profesionales. Por otra parte, y de acuerdo con las características de nuestro sistema educativo, constituyen un condicionante de acceso a otros niveles del sistema y tipos de estudios. 
Construcción y validación del Cuestionario de Preferencias Profesionales (CPP). El CPP tiene como marco teórico de referencia los "intereses básicos" y la línea trazada por las investigaciones anglosajonas y su literatura científica sobre escalas de intereses profesionales desde el Vocational Interest Blank (Strong, 1927), hasta sus últimas revisiones y adaptaciones (Campbell, 1971; Hansen and Campbell, 1985; Harmon et al., 1994). El CPP contempla 17 áreas vocacionales de intereses profesionales básicos en 182 items con cinco posibilidades de respuesta (Likert), en los que el encuestado expresa su agrado o desagrado con las profesiones y actividades profesionales propuestas, con lo que se pretende jerarquizar las preferencias o intereses personales de las 17 áreas vocacionales que presenta el cuestionario. La selección de las 17 áreas vocacionales del CPP se ha llevado a cabo teniendo en cuenta algunos de los principales trabajos sobre el tema realizados en España, dada la fiabilidad y consistencia contrastada de los instrumentos utilizados para el estudio de los intereses profesionales de los alumnos de secundaria (Yuste, 1988; García Mediavilla et al. 1990; De la Cruz, 1997; Rivas, et al. 1998; Hernández Franco, 2004). El CPP puede considerarse como un cuestionario de autopercepción de intereses expresados que integra items correspondientes a 17 áreas vocacionales. En el tratamiento de los datos y su análisis crítico y estadístico se ha utilizado el paquete estadístico SPSS 11.00 para Windows (Pardo y Ruiz, 2002) y la hoja de cálculo Excel 2003 (Valdés-Miranda y Rodríguez, 2004). Los datos recogidos tras su aplicación experimental fueron sometidos a un análisis factorial por el método de extracción de componentes principales y de rotación por normalización Varimax con Kaiser, mientras que su consistencia interna y la de las 17 escalas vocacionales que lo componen se determinó mediante el cálculo del coeficiente $\alpha$ de Cronbach, cuyos resultados presentamos en la tabla 1.

Adoptando el criterio de que el coeficiente $\alpha$ de Cronbach sea superior a 0.800 (Gingres, 1990), todos los coeficientes obtenidos, tanto para el CCP (0.967) como para sus escalas vocacionales son superiores a dicho valor, exceptuando la escala 13 (Comunicación, $\alpha=0.774$ ), lo que nos indica que el cuestionario presenta una alta consistencia que certifica su validez interna y nos garantiza que los perfiles obtenidos, a partir de las puntuaciones alcanzadas por un sujeto, nos muestran las preferencias relativas de un área respecto a las restantes y permite que el alumno identifique su primera y segunda áreas vocacionales de interés preferente, en un proceso subjetivo de autoexploración de sus cogniciones vocacionales.

Construcción y validación del Cuestionario de condicionantes de la toma de decisiones $(\boldsymbol{Q})$. El cuestionario Q, con sus 35 items, es el resultado de la síntesis de una batería de siete cuestionarios de 30 items cada uno (total 210 items), de los que por razones de funcionalidad y economía de tiempos, se seleccionaron, después de la prueba piloto, los 5 más significativos de cada cuestionario con los que elaboramos el cuestionario experimental que nos aporta información sensible sobre las siguientes siete condicionantes de la toma de decisiones:

1) Económicos.

2) Familiares.

3) Geográficos.

4) De la salud.

5) Personales. 
TABLA 1

\begin{tabular}{|l|l|c|c|}
\hline \multicolumn{1}{|c|}{ Escalas } & \multicolumn{1}{|c|}{ Items $\mathbf{n}^{\mathbf{0}}$} & $\begin{array}{c}\text { Total } \\
\text { items. }\end{array}$ & Coef. $\boldsymbol{\alpha}$ \\
\hline 1. Científica & $6,16,23,41,83,97,105,144,153$ y 163 & 10 & 0.833 \\
\hline 2. Técnica & $21,28,32,34,43,48,60,62,73,94$ y 168 & 11 & 0.886 \\
\hline 3. Económico-empresarial & $56,58,63,64,86,106,112,114,118,122$ y 147 & 11 & 0.833 \\
\hline 4. Ciencias de la naturaleza & $1,3,7,71,78,90,135,152,159,174$ y 178 & 11 & 0.858 \\
\hline 5. Agropecuaria & $9,14,18,38,109,123,124,155,161,181$ y 182 & 11 & 0.892 \\
\hline 6. Biosanitaria & $2,5,15,30,37,68,69,89,102$ y 173 & 10 & 0.920 \\
\hline 7. Ocio & $11,12,26,29,51,59,74,77,81,148$ y 156 & 11 & 0.835 \\
\hline 8. Profesionales del deporte & $24,31,40,53,75,80,108,162,164$ y 169 & 10 & 0.954 \\
\hline 9. Humanidades & $47,66,72,88,95,104,115,127,137,149$ y 180 & 11 & 0.818 \\
\hline 10. Psicopedagógica & $39,42,61,67,87,111,117,129,133$ y 139 & 10 & 0.872 \\
\hline 11. Sociológica & $46,65,70,91,128,131,140,141,150,151$ y 175 & 11 & 0.863 \\
\hline 12. Derecho y asesoramiento & $17,20,25,50,79,98,116,134,136,157$ y 170 & 11 & 0.885 \\
\hline 13. Comunicación & $4,8,10,35,57,130,132,145,160,165$ y 171 & 11 & 0.774 \\
\hline 14. Seguridad & $27,45,54,93,103,121,142,146,172,176$ y 179 & 11 & 0.869 \\
\hline 15. Artes escénicas & $13,33,85,92,99,107,120,125,154,158$ y 166 & 11 & 0.896 \\
\hline 16. Música & $19,22,44,84,96,113,126,143,167$ y 177 & 10 & 0.900 \\
\hline 17. Artes plásticas & $36,49,52,55,76,82,100,101,110,119$ y 138 & 11 & 0.896 \\
\hline Cuestionario CPP & & 182 & $\mathbf{0}, 967$ \\
\hline
\end{tabular}

6) Hábitos de estudio y

7) Rendimiento académico.

En el tratamiento de los datos y su análisis crítico y estadístico se ha utilizado el paquete estadístico SPSS 11.00 para Windows (Pardo y Ruiz, 2002) y la hoja de cálculo Excel 2003 (Valdés-Miranda y Rodríguez, 2004). Los datos recogidos tras su aplicación experimental fueron sometidos a un análisis factorial por el método de extracción de componentes principales y de rotación por normalización Varimax con Kaiser, mientras que su consistencia interna y la de las 7 escalas que lo componen se determinó mediante el cálculo del coeficiente a de Cronbach, cuyos resultados presentamos en la tabla 2.

El coeficiente $\alpha$ de Cronbach (0.611) del cuestionario Q es bajo, lo que nos indica una deficiente consistencia interna del mismo, debido lógicamente a la diversidad temática de las 7 escalas que lo componen y que nos exige posteriores revisiones para su mejora. Para 
TABLA 2

\begin{tabular}{|l|l|c|c|}
\hline \multicolumn{1}{|c|}{ Escalas } & \multicolumn{1}{c|}{ Items $\mathbf{n}^{\mathbf{0}}$} & Total items. & Coef. $\boldsymbol{\alpha}$ \\
\hline 1. C. económicos & $5,8,15,29$ y 32 & 5 & 0.777 \\
\hline 2. C. familiares & $6,11,14,25$, y 27 & 5 & 0.689 \\
\hline 3. C. geográficos & $1,20,23,33$ y 35 & 5 & 0.869 \\
\hline 4. C. de la salud & $2,13,18,22$ y 30 & 5 & 0.575 \\
\hline 5. C. Personales & $10,16,21,26$ y 34 & 5 & 0.617 \\
\hline 6. C. hábitos de estudio & $4,12,19,28$ y 31 & 5 & 0.769 \\
\hline 7. C. rendimiento académico & $3,7,9,17$ y 24 & 5 & 0.534 \\
\hline Cuestionario $\mathbf{Q}$ & & $\mathbf{3 5}$ & $\mathbf{0 . 6 1 1}$ \\
\hline
\end{tabular}

paliar esta deficiencia en una primera corrección aglutinamos los items de las 7 escalas en 3 dimensiones:

A) Condicionantes generales (Escalas 1, 2, 3 y 4).

B) Condicionantes personales (Escala 5) y

C) Condicionantes del estudio (Escalas 6 y 7).

Los resultados obtenidos tras su análisis factorial se presentan en la tabla 3.

TABLA 3

\begin{tabular}{|c|c|c|c|}
\hline Dimensiones & Items $\mathrm{n}^{\circ}$ & $\begin{array}{l}\text { Total } \\
\text { items. }\end{array}$ & $\begin{array}{l}\text { Coef. } \\
a\end{array}$ \\
\hline 1. Condicionantes generales & $\begin{array}{l}1,2,5,6,8,11,13,14,15,18,20,22,23,25 \\
27,29,30,32 ., 33, \text { у } 35\end{array}$ & 20 & 0.812 \\
\hline 2. Condicionantes personales & $10,16,21,26$ y 34 & 5 & 0.617 \\
\hline 3. Condicionantes del estudio & $3,4,7,9,12,17,19,24,28$ у 31 & 10 & 0.799 \\
\hline Cuestionario Q & & 35 & 0.752 \\
\hline
\end{tabular}

Tras esta corrección el coeficiente $\alpha$ de Cronbach para el cuestionario Q es de 0.752 , y aunque el grado de consistencia es bastante aceptable y nos permite utilizarlo, recomendamos una cuidadosa revisión de sus items para mejorar la consistencia interna de sus escalas y dar una mayor fiabilidad al instrumento.

Validación externa del METODE.Bach. Se han construído y validado tres cuestionarios (alumnos, padres y tutores/orientadores) para determinar el grado de satisfacción manifestado tras la aplicación de METODE.Bach y la toma de decisiones para la validación externa del Método . Los tres cuestionarios fueron adaptados y validados para esta investigación partiendo de trabajos anteriores en los que se contemplan 4 dimensiones básicas para 
evaluar la satisfacción de los participantes tras la aplicación del Método y la correspondiente toma de decisiones (Benavent el al., 2001 y 2002):

1) Utilidad funcional y eficacia del Método.

2) Pertinencia de su diseño, formato y estructura.

3) Aporta información precisa y valiosa y

4) Facilita una adecuada toma de decisiones.

En la tabla 4 se presenta un resumen de los resultados que justifican la validez externa del Método:

TABLA 4

\begin{tabular}{|l|c|c|c|c|}
\hline \multicolumn{1}{|c|}{ Cuestionario } & $\begin{array}{c}\mathbf{N}^{\circ} \\
\text { sujetos }\end{array}$ & $\begin{array}{c}\mathbf{N}^{\circ} \\
\text { items }\end{array}$ & $\begin{array}{c}\text { Coef. } \\
\boldsymbol{\alpha}\end{array}$ & Grado de satisfacción expresado \\
\hline Para los alumnos & 372 & 20 & 0.822 & $\begin{array}{c}3.006+0.030 \text { (satisfecho). } \\
(\mathrm{gl}=3 ; \mathrm{M}=3.006 ; \quad \sigma=0.372 ; \sigma \mathrm{M}=0.030)\end{array}$ \\
\hline Para los padres & 206 & 14 & 0.865 & $\begin{array}{c}2.986+0.035 \text { (satisfecho). } \\
(\mathrm{gl}=3 ; \mathrm{M}=2.986 ; \quad \sigma=0.515 ; \sigma \mathrm{M}=0.035)\end{array}$ \\
\hline Para el tutor/orientador & 19 & 12 & 0.733 & $\begin{array}{c}3.807+0.077 \text { (muy satisfecho). } \\
(\mathrm{gl}=3 ; \mathrm{M}=3.807 ; \quad \sigma=0.335 ; \quad \sigma \mathrm{M}=0.077)\end{array}$ \\
\hline
\end{tabular}

Con los resultados obtenidos, consideramos que hemos alcanzado los objetivos propuestos al construir y validar un método sencillo, económico y eficaz para orientar a los alumnos de Bachillerato y sus familias en la toma de sus decisiones académicas y profesionales al finalizar su formación en la educación secundaria.

\section{Conclusiones}

METODE.Bach: Método para la toma de decisiones al finalizar el Bachillerato (Benavent et al., 2007), se presenta en formato texto en un cuadernillo autoaplicable (papel y lápiz) de 98 páginas. Además, se ha diseñado un programa original interactivo en CD-Rom para ordenador personal, a fin de que el alumno lo pueda utilizar de modo autónomo e individual en el centro escolar o en el hogar.

El cuadernillo del METODE.Bach en formato texto autoaplicable, versión "papel y lápiz", incluye distintos instrumentos y presenta la siguiente estructura: Índice. Datos personales. Introducción. $1^{\text {a }}$ Parte: Resultados académicos (rejillas autoaplicables con escala de valoración de resultados). $2^{\text {a }}$ Parte: Aplicación de los cuestionarios y valoración de los resultados (cuestionarios CPP y Q, hoja de respuestas, registro de datos, ajuste de puntuaciones y escalas de valoración). $3^{\text {a }}$ Parte: Proceso de toma de decisiones y consejo orientador (distintas estrategias para la combinación de los resultados de los instrumentos utilizados que facilitan y conducen a la toma de decisiones). 4 ${ }^{\mathrm{a}}$ Parte: Áreas vocacionales y niveles de opción (información para utilizar en el proceso). Anexos: Otros estudios y direcciones postales, electrónicas y telemáticas de interés (información complementaria y de ampliación). 
El Método culmina con la toma de decisiones y la formulación del correspondiente consejo orientador. La decisión final la alcanza el alumno utilizando todos los datos acumulados y arrastrados a lo largo del proceso, información que es tratada por medio de originales y sencillas estrategias de operativa combinatoria propias del Método. Las puntuaciones cuantitativas se transforman en escalas cualitativas de cinco escalones, que permiten un cotejo binario de los condicionantes, las calificaciones escolares y las opciones académicas y/o laborales en función de las áreas vocaciones preferentes, por medio de la elaboración de tablas de equivalencia de doble entrada y la auto-asignación de un número clave, indicador del grado de libertad de la elección en cada caso. Si el número clave que se auto-asigna el sujeto, le indica que su elección es totalmente dependiente, es decir, que carece del grado mínimo de libertad para elegir responsablemente y con garantías de éxito, se interrumpe el proceso, no se le permite la toma de una decisión final y se le recomienda hablar con el orientador/a o tutor/a de su centro educativo.

Por último, deseamos constatar que METODE.Bach, es el resultado de una rigurosa y laboriosa investigación aplicada, tan oportuna como necesaria, que contribuirá de modo positivo a la orientación académica y profesional de las poblaciones escolares que están cursando el Bachillerato, para facilitarles la toma de decisiones al finalizar su educación secundaria post-obligatoria. Aunque estamos convencidos de que nuestro Método autoaplicable, facilitará la tarea de los profesores tutores y orientadores en la formulación del consejo orientador al finalizar el Bachillerato, consideramos conveniente, por haber sido construido en el ámbito geográfico de la Comunidad Valenciana, su contraste y validación en las restantes CC. AA. de España. De este modo, en una segunda fase y con la colaboración de todos los profesionales de la orientación que lo utilicen, queda abierta la posibilidad de elaborar baremos, a partir de perfiles empíricos procedentes de grupos normativos, que permitan las comparaciones entre los resultados obtenidos en las distintas CC. AA., así como su pulido, adaptación y traducción a todas las lenguas oficiales del Estado Español, a fin de mejorar su adecuación a los diferentes marcos socio-culturales y optimizar su eficacia.

\section{Referencias Bibliográficas}

Álvarez Rojo, V. (1991). "Tengo que decidirme". 2 vol.: Cuaderno del tutor y Cuaderno del alumno. Sevilla: Edit. Alfar.

Álvarez, M. y Fernández, R. (1987). Programa de orientación de estudios y vocacional al término de la escolaridad obligatoria. ( $5^{\mathrm{a}}$ ed. revisada 1996), Barcelona: PPU.

Benavent, J. A. (1994). "Un modelo cognitivo base para la elaboración de programas de Orientación para el desarrollo de la carrera". En AEOEP, Desarrollo de la carrera: Modelos y programas actuales (pp. 37-43). Valencia: Asociación Española de Orientación Escolar y Profesional. Delegación de Valencia.

Benavent, J. A.; Bayarri, F.; García, J.; Ramírez, L. y Vivo, S. (2000). "Programa de autoayuda para la toma de decisiones al finalizar la ESO (CESOF)". 2 vol.: Cuaderno del Orientador-tutor y Cuaderno del alumno. Valencia: Departamento de Didáctica y Organización Escolar. Universidad de Valencia.

Benavent, J. A.; Bayarri, F.; García, J.; Ramírez, L. y Vivo, S. (2000a). Programa de autoayuda para la toma de decisiones al finalizar la ESO (CESOF). Versión de aplicación: CD-Rom interactivo. Valencia: Macromedia Inc.-SiCon. 
Benavent, J. A., Bayarri, F., García, J., Ramírez, L. y Vivo, S. (2001). "Programa de autoayuda para la toma de decisiones al finalizar la Educación Secundaria Obligatoria (ESO). CESOF/06". Revista Española de Orientación y Psicopedagogía, 12 (22): 283-302.

Benavent, J. A.; Bayarri, F.; García, J.; Ramírez, L. y Vivo, S. (2002). "Programa de autoayuda para la toma de decisiones al finalizar la Educación Secundaria Obligatoria (ESO). CESOF/06". En Álvarez, M. y Bisquerra, R. (Eds.), Manual de Orientación y Tutoría (pp. 330/275-330/301). Vol. II. Barcelona: Editorial CISS-Praxis.

Benavent, J. A.; Bayarri, F.; García, J.; Ramírez, L. y Vivo, S. (2007). METODE.Bach: Método para la toma de decisiones al finalizar el Bachillerato. Valencia: Edit. Tirant lo Blanch.

Benavent, J. A.; Bayarri, F.; García, J.; Ramírez, L. y Vivo, S. (2007). METODE.Bach: Método para la toma de decisiones al finalizar el Bachillerato. Versión de aplicación: CD-Rom interactivo. Valencia: Edit. Tirant lo Blanch.

Bisquerra, R. et al. (1989). Quadern d'orientació als estudiants. Barcelona: Generalitat de Catalunya.

Cambell, D. P. (1971). Handbook for the Strong Interest Vocational Blank. Stanford, C.A.: Stanford University Press.

Corominas, E, et al. (1999). "Programa de Orientación Profesional Autoaplicado (POPA)". En Álvarez M. y Bisquerra, R. (Eds.), Manual de Orientación y Tutoría (pp. 330/155-330/176). Barcelona: Edit. Praxis.

De la Cruz, M ${ }^{\mathrm{a}}$ V. (1993). IPP Inventario de Intereses y Preferencias Profesionales: Manual. (3 ${ }^{\mathrm{a}}$ ed. revisada 1997). Madrid: TEA Ediciones.

Duchastel, P. (1986). "Intelligent computer assisted instruction system: The natur of learner control". Journal of Educational Computing Research, 2 (3): 379-393.

García Mediavilla, L.; Izquierdo, R. y Sánchez-Cabezudo, J. (1990). El test "CIP-II”. Como descubrir los intereses profesionales. Madrid: Edit. Bruño.

Gingres, M. (1990). Elaboration d'une stratégie d'evaluation des besoins d'education à la carrière chez la finissants du secondaire. Tesis doctoral. Sherbrooke: Université de Sherbrooke.

Hansen, J. C. and Campbell, D. P. (1985). Manual for the SVIB-SCII: Strong-Campbell Interest Inventory. Palo Alto, C.A.: Consulting Psychologist Press.

Harmon, L. W.; Hansen, J. C.; Borgen, F. H. and Hammer, A. L. (1994). Strong Interest Inventory. Applications and Technical Guide. Stanford, C.A.: Stanford University Press.

Hernández Franco, V. (2004). "Evaluación de los intereses básicos académico profesionales de los estudiantes de secundaria". Revista Española de Orientación y Psoicopedagogía, 15 (1): 117-141.

Hernández Franco, V.; Muñoz San Roque, I.; Asensio, C. y Caro, C. (2004). Ciclos formativos. Preferencias de los estudiantes de la Comunidad de Madrid. Madrid: Universidad Pontificia de Comillas. Servicio de Publicaciones.

Martínez, J. M. y Valls, F. (1999). "Estudio preliminar para la adaptación del programa autoaplicable de elección vocacional 'Self-Directed Search de John L. Holland' '. Revista Española de Orientación y Psicopedagogía, 10 (18): 353-368.

MEC (2006). Ley Orgánica de Educación. Madrid: Autor. Secretaría de Publicaciones. También disponible en: http://www.mec.es (2006, 20 de septiembre).

Pardo, A. y Ruiz, M. A. (2002). SPSS 11: Guía para el análisis de datos. Madrid: McGraw-Hill Interamericana de España.

Peterson, G. W.; Sampson, J. P. and Reardom, R. C. (1991). Career Development and Services: A Congnitive Approach. Pacific Grove, CA: Brooks/Cole.

Repetto, E. (1999). Tu futuro profesional. 5 vol.: Libro del Profesor-tutor, 2 vol. y Libro del alumno, 3 vol. Madrid: Edit. CEPE S. L. 
Rivas, F. et al. (1989). Sistema de Autoayuda Vocacional: SAV. Valencia: Consejería de Cultura, Educación y Ciencia.

Rivas, F. et al. (1998). Manual del SAV-R y SAVI-2000. Sistemas de Autoayuda y Asesoramiento Vocacional. (Manual, carpeta de materiales y CD-Rom interactivo). Valencia: Servicio de Asesoramiento Vocacional y Educativo S. L.

Rodríguez Moreno, Mª. L. (1994). Programa para enseñar a tomar decisiones. Barcelona: Edit. Laertes.

Strong, E. K. (1927). Vocational Interest Blank. Stanford, C. A.: Stanford University Press.

Valdés-Miranda, C. y Rodríguez Álvarez, E. (2004). Manual imprescindible de Excel 2003. Madrid: Anaya Multimedia.

Yuste, C. (1988). Preferencias profesionales. 2 vol.: Manual técnico: nivel medio y Manual técnico: nivel superior. Madrid: CEPE. (Yuste, C y Galve, J. L. Edición revisada y renovada, 2001).

Fecha finalización: diciembre de 2007

Fecha de recepción: 20-12-2007

Fecha de revisión: 19-11-2008

Fecha de aceptación: 05-05-2009 